\title{
A Smart Vertical Handoff Decision Algorithm Based on Queuing Theory
}

\author{
Yong Sun*, Chao Liu**, Peng Yang**, Xiangming Wen* \\ *Beijing Key Laboratory of Network System Architecture and Convergence \\ **Beijing University of Posts and Telecommunications, Beijing, China 100876 \\ sunyong@bupt.edu.cn, 2011110249@bupt.edu.cn
}

\begin{abstract}
Vertical handoff is crucial to future wireless communication and keeps challenging. In this paper, a smart vertical handoff decision algorithm based on queuing theory is proposed. This algorithm formulates the whole heterogeneous wireless area and handoff procedure using queuing theory and proposes a new network selection index called new handoff blocking probability to evaluate the network performance. We also regulate the network architecture to manage the wireless resource effectively. The experimental results show that the proposed algorithm outperforms the traditional algorithm with low handoff blocking probability and a better load balance of the whole wireless environment.
\end{abstract}

Keywords - Wireless resource management, Vertical handoff, Network selection, Queuing theory, Heterogeneous wireless networks

\section{INTRODUCTION}

The fast evolution of mobile communications results in an increasing number of heterogeneous networks. As can be predicted, heterogeneous wireless networks with various technologies and standards will coexist for a long period of time, determine their own market positions according to the principles of market competition and complementation and gradually become converged rather than unified on a platform of next-generation networks [1]. Facing this problem, the architecture for the Beyond 3rd Generation (B3G) or 4th Generation $(4 \mathrm{G})$ wireless networks aims to integrate various heterogeneous wireless access networks over an IP backbone [2].

To reach this goal, seamless mobility support in a heterogeneous roaming environment is one of the most challenging issues [3]. In heterogeneous wireless networks, seamless handoff can be divided into two categories: horizontal handoff (HHO) and vertical handoff (VHO) [4]. Compared with the horizontal handoff, the vertical handoff (handoff between networks interfaces with different link-layer technologies) is more valuable for research. Specially, in seamless vertical handoff process, handoff decision is an important and intelligent part, which is used to determine whether and how to perform the handoff by evaluating and selecting the most appropriate access network [5]. The main problem for vertical handoff decision is how the handoff strategy regulates network selection so as to make a good trade-off between the performance optimization of single $\mathrm{MN}$ and efficient utilization of whole wireless environment.

The rest of the paper is organized as follows: In Sect. II, we briefly review the related works about vertical handoff decision algorithms. In Sect. III, a smart vertical handoff decision algorithm based on queuing theory is proposed. Sect. IV shows the performance evaluation of our proposed algorithm. Finally, a conclusion is summarized in Sect. V.

\section{RELATED WORKS}

Many handoff algorithms have been proposed and the main methods can be summarized as following. (1) Fuzzy logic, fuzzy logic is proposed to represent the imprecise information of the conditions about the heterogeneous networks and to adapt dynamically to evaluate multiple attributes simultaneously [6]. (2) AHP (Analytic Hierarchy Process), AHP decomposes the network selection problem into several sub-problems and assigns a weight value to each sub-problem [7]. Then, the network with the highest performance score is selected. (3) SSF (Strongest Signal First), in this model RSS is the only affecting factor of selecting access network.

Although there are various vertical handoff algorithms, some problems remain unsolved. Some algorithms, such as $\mathrm{SSF}$, tend to adopt a simple decision algorithm to maintain a faster handoff procedure. But the simple decision-making mechanism may not help to select a suitable network and may also yield serious Ping-Pong effect. Others regard the handoff decision procedure as a multiple attribute decision making (MADM) problem [8-10, 15] and tend to solve the problem by searching for the optimal solution. However, these algorithms only emphasize on the optimized network for single $\mathrm{MN}$ (mobile node) and neglect the evaluation of the whole wireless environment, which may cause the unbalanced loads distribution, serious time delay and the increasing number of handoff dropping. To overcome these problems, our algorithm is proposed. In this paper we formulate the whole heterogeneous wireless environment and handoff procedure using queuing theory. We also regulate the network architecture to manage the wireless resource effectively. Then, we propose a new network selection index called new handoff blocking probability to evaluate the network performance. Finally a smart vertical handoff decision algorithm based on queuing theory is proposed. 


\section{III.THE Proposed SCHEME}

In heterogeneous wireless networks, the coverage environment can be divided into two areas: cellular coverage area and WLAN hotspots area. The WLANs are typically configured as small cells within the "cellular coverage area" of GPRS/UMTS or CDMA2000, which is relatively larger compared with WLAN hotpots [11]. The cellular coverage area is covered by a set of overlapping BSs $B=\left\{b_{1}, b_{2} \cdots b_{M}\right\}$ and a set of WLAN APs $A=\left\{a_{1}, a_{2} \cdots a_{N}\right\}$. In our algorithm, the cellular coverage area is implemented in a vertical handoff decision system (VHDS). The VHDS consists of two parts: multiple vertical handoff decision transducers (VHDT) and a central vertical handoff decision controller (CVHDC). VHDTs are located in each access network in order to provide real-time network conditions and handoff requests of networks in the cellular area. CVHDC execute the vertical handoff decision for the whole wireless region after analyzing the collected message from VHDTs. The signaling interaction between CVHDC, VHDT and access networks will be obtainable by the media independent handoff (MIH) which is defined in IEEE 802.21 [12].

VHDS maintains the sets $A$ and $B$ which covers the cellular coverage region as a list $S$ of candidate access points. All available WLAN APs in set $A$ and BS in set $B$ are added into $S$. In addition, we define $U=\left\{u_{1}, u_{2} \cdots u_{K}\right\}$ as the set of all the mobile nodes $(\mathrm{MN})$ which have the probability to access the network in the cellular coverage region. Only these $K \mathrm{MNs}$ are considered in this model and each $\mathrm{MN}$ is either requesting a handoff (or just turned on and require channel) or remains connected to an $\mathrm{AP}(\in A)$ or $\mathrm{BS}(\in B)$. Then, we divide the set $U$ into two subsets at a certain time: $U_{h}(t)$ and $U_{r}(t)$.

$$
U_{h}(t)=\left\{u_{h 1}, u_{h 2} \cdots u_{h(t)}\right\}
$$

In equation (1), $U_{h}(t)$ stands for the set of MNs which request handoff at the certain time $t$ and $h(t)$ is the number of $\operatorname{set} U_{h}(t)$.

$$
U_{r}(t)=U-U_{h}(t)=\left\{u_{r 1}, u_{r 2} \cdots u_{r(t)}\right\}
$$

Accordingly, $U_{r}(t)$ is the complementary set of $U_{r}(t)$ and represents the MNs which will remain the connection to the current networks at the certain time. $r(t)$ is the number of set $U_{r}(t)$.

The bandwidth change problem can be formulated by queuing theory. It is noted that the vertical handoff decision algorithm is deployed in each AP or BS. And each AP or BS has the limitation to the available bandwidth which means that the channels provided by the network is limited by each network and only certain number of connection can be maintained. To simplify the problem, we assume that only $L a$ channels and $L b$ channels are allocated by each AP or BS respectively. Fig.2 shows the decision-making epochs of CVHDC.

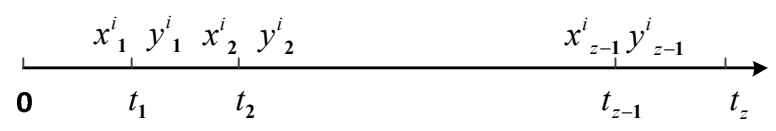

Figure 1. Successive time epochs of network

The sequence $T=\left\{t_{1}, t_{2} \cdots t_{z}\right\}$ represents successive time epochs, where the variable $t_{z}$ denotes the current time epoch. For each network $i$, the handoff arrival rate sequence $X_{i}=\left\{x_{1}^{i}, x_{2}^{i} \cdots x_{z-1}^{i}\right\}$ and the service complete rate sequence $Y_{i}=\left\{y_{1}^{i}, y_{2}^{i} \cdots y_{z-1}^{i}\right\}$ will be stored and CVHDC will make the decision at $t_{z}$ based on them.

For the traffic characteristics, the handoff decision and channel allocation process of a network $a_{i}$ or $b_{i}$ can be formulated as $M / M / n / n$ model. To dynamically adjust the process of network selection, we make the variable $n$ dynamically respect the available channels $L a_{(a v)}$ and $L b_{(a v)}$ which are not occupied by $\mathrm{MNs}$ for $\mathrm{AP}$ and $\mathrm{BS}$, respectively. The arrival of handoff requests for channels follows a Poisson distribution with mean $\lambda_{i}$ and the service holding time is assumed in a negative exponential distribution with mean $1 / \mu_{i}$ for each network $i$. These assumptions are common in the many researchers $[13,14]$. However, in our model we will dynamically adjust the $\lambda_{i}$ and $\mu_{i}$ according to the real-time network condition sequences $X_{i}$ and $Y_{i}$ which is stored in CVHDC. So $\lambda_{i}$ and $\mu_{i}$ will change in each time epoch and can be redefined to $\lambda_{i}\left(t_{z-1}\right)$ and $\mu_{i}\left(t_{z-1}\right)$ as function (3) and (4).

$$
\begin{aligned}
& \lambda_{i}\left(t_{z-1}\right)=\left(1 / t_{z-1}\right) \sum_{k=1}^{z-1} x_{k} \\
& \mu_{i}\left(t_{z-1}\right)=\left(1 / t_{z-1}\right) \sum_{k=1}^{z-1} y_{k}
\end{aligned}
$$

Note that the network in the cellular coverage area does not have holding queue, so the handoff will be blocked if all $n$ channels of target network is occupied. Thus, we can define the probability which the network $i$ is fully occupied as the new handoff blocking probability (NHBP) $P_{i}$ which is derived from the new call blocking probability. $P_{i}$ is defined according to the queuing theory as function (5). And we also propose the max blocking probability of the entire area by function (6).

$$
\begin{gathered}
P_{i}=\left(\lambda_{i}\left(t_{z-1}\right) / \mu_{i}\left(t_{z-1}\right)\right)^{n}\left(n ! \sum_{j=0}^{n}\left(\left(\lambda_{i}\left(t_{z-1}\right) / \mu_{i}\left(t_{z-1}\right)\right)^{j} / j !\right)\right)^{-1} \\
P_{b}=M A X_{1 \leq i \leq M+N}\left(P_{i}(E)\right)
\end{gathered}
$$

The variable $P_{b}$ is the max blocking probability of the entire cellular coverage area before the CVHDC makes the handoff decision at $t_{z}$. And our algorithm is aiming at minimizing the max blocking probability of the entire cellular coverage area. 
To describe the connection status between the MNs $(\in U)$ and the networks $(\in A \cup B)$, an association matrix $C(t)=\left\{c_{i j}\right\}_{(M+N) \times K}$ for the cellular coverage area is proposed. $c_{i j}$ is equal to 1 only when there is a connection between network $a_{i}$ or $b_{i}$ and the $\mathrm{MN} u_{j}$ and 0 otherwise.

We also define the possible association matrix at $t_{z}$ as

$$
\begin{aligned}
C^{\prime}\left(t_{z}\right)= & \left\{c^{\prime}{ }_{i j}\right\}_{(M+N) \times K}: \\
& c^{\prime}{ }_{i j}=\{0,1\} \\
& c^{\prime}{ }_{i j}=0 \quad R S S_{i j}< \begin{cases}\theta_{a} & \text { for } 1 \leq i \leq N \\
\theta_{b} & \text { for } N+1 \leq i \leq N+M\end{cases} \\
& \sum_{i=1}^{N+M} c^{\prime}{ }_{i j}^{\prime}=1
\end{aligned}
$$

In the possible association matrix $C^{\prime}\left(t_{z}\right), c_{i j}^{\prime}$ is defined as 1 in two situations. One is that the $\mathrm{MN} u_{j}$ remains connected to the current network $a_{i}$ or $b_{i}$. Another is that the MN $u_{j}$ will hands off to the candidate network $a_{i}$ or $b_{i}$. Note that each $\mathrm{MN}$ in the set $U_{h}(t)$ may have multiple candidate networks which satisfy the RSS thresholds $\theta_{a}$ (for AP) or $\theta_{b}$ (for BS), so the possible association matrix may also be multiple. Accordingly, we define $\chi\left(t_{z}\right)$ as the set of possible association matrices of $t_{z}$. For each possible association matrix $C^{\prime}\left(t_{z}\right)$ in $\chi\left(t_{z}\right)$, we will generate a handoff matrix by the function (10):

$$
H\left(t_{z}\right)=C^{\prime}\left(t_{z}\right)-C\left(t_{z}\right)=\left\{H_{i j}\right\}_{(M+N) \times K}
$$

$H\left(t_{z}\right)$ denotes the originate network $i$ and the target network $j$ of the handoff of MN $u_{k}$ with the value $h_{i k}=-1$ and $h_{j k}=1 . h_{\alpha \beta}$ is equal to 0 only when the $\mathrm{MN} u_{\beta}$ remains the current connection. For the certain network $i$, we calculate the number of -1 and 1 in the $i$ row of $H\left(t_{z}\right)$ as $x_{\text {off }}$ and $x_{i n}$, which denotes the number of MNs leaving and accessing the network. Then the model can be reformulated as following. For each network $i$ :

$$
\begin{gathered}
\lambda_{i}\left(t_{z}\right)=\left(1 / t_{z}\right)\left(\sum_{k=1}^{z-1} x_{k}+x_{i n}\right) \\
\mu_{i}\left(t_{z}\right)=\left(1 / t_{z}\right)\left(\sum_{k=1}^{z-1} y_{k}+x_{o f f}\right) \\
P_{i}(\mathrm{E})=\left(\lambda_{i}\left(t_{z}\right) / \mu_{i}\left(t_{z}\right)\right)^{n}\left(n ! \sum_{j=0}^{n}\left(\left(\lambda_{i}\left(t_{z}\right) / \mu_{i}\left(t_{z}\right)\right)^{j} / j !\right)\right)^{-1}
\end{gathered}
$$

And for any possible association matrix $C^{\prime}\left(t_{z}\right) \in \chi\left(t_{z}\right)$

$$
P_{b}\left(C^{\prime}\left(t_{z}\right)\right)=M A X_{1 \leq i \leq M+N}\left(P_{i}(E)\right)
$$

Thus an algorithm aimed at minimizing expected new handoff blocking probability is proposed as follows:

Min-P: $\operatorname{Min}_{\forall C^{\prime}\left(t_{z}\right) \in \chi\left(t_{z}\right)}\left(M A X_{1 \leq i \leq M+N}\left(P_{i}(E)\right)\right.$

\section{IV.SIMULATION RESULTS AND ANALYSIS}

In this section, our algorithm is evaluated by simulation compared with the AHP algorithm and SSF (Strong-Signal First). To ease our illustration, we just consider the situation in which there are two kinds of networks $3 \mathrm{G}$ and $802.11 \mathrm{~b}$. And the above two integrated heterogeneous wireless networks represent the set $B$ and the set $A$ in our algorithm respectively. The simulation topologies of heterogeneous wireless networks are shown in Fig.2. The cellular coverage area is covered by two overlapping $3 \mathrm{G} \mathrm{BSs}$ and four $802.11 \mathrm{~b}$ hotpots. The maximum bandwidth of BSs and APs are predefined as $3 \mathrm{mbps}$ and $7 \mathrm{mbps}$, respectively. Besides, the bandwidth requested by each $\mathrm{MN}$ is constant $0.2 \mathrm{mbps}$. So according to the service request of $\mathrm{MN}$, we can divide the $\mathrm{BS}$ and $\mathrm{AP}$ in 15 and 35 Channels. Then the model can be formulated as $M / M / 35 / 35$ and $M / M / 15 / 15$ for each BS and AP.

At the beginning of the simulation, MNs are evenly distributed over the coverage area. The MNs move around during the entire simulation time. We utilize a random mobility method to characterize the movement of MNs in the coverage environment. All the attributes (including RSS) for each MN and AP or BS association are reselected after each such movement. For simplicity, it is assumed in the simulations that each BS or AP will satisfy the MN's RSS threshold if it is within the coverage area of that BS or AP.

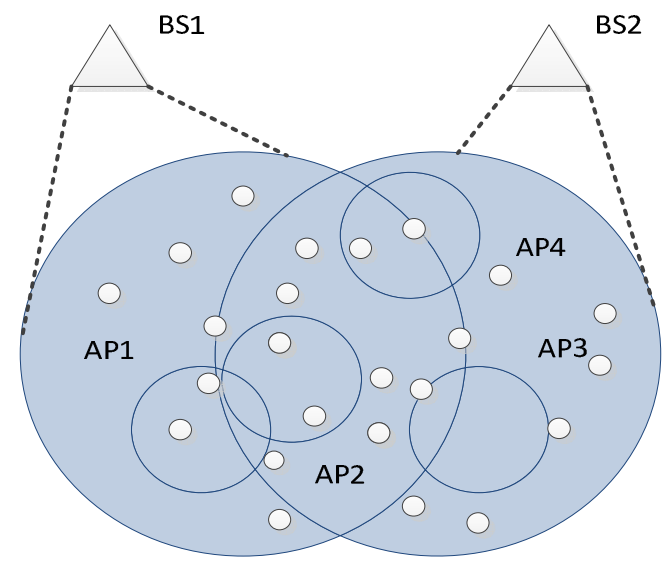

Figure 2. Simulation topologies of heterogeneous networks

We evaluate the load balancing performance of our algorithm, the AHP algorithm and the SSF (Strong-Signal First) method. The lower the value is the better performance of load balancing the algorithm gets. Two independent simulations run with a duration of 1000s with the number of MNs 100 and 150, respectively. 


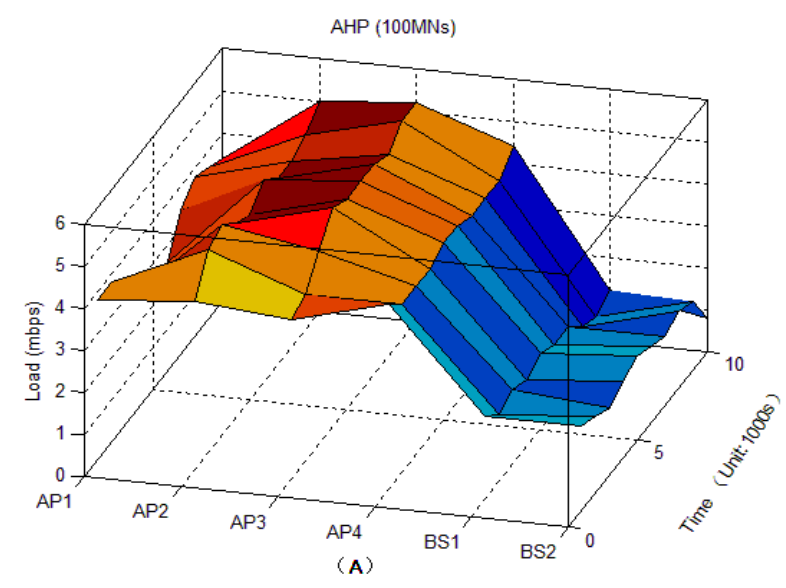

Figure 3. Load status for the AHP algorithm, when there are $100 \mathrm{MNs}$

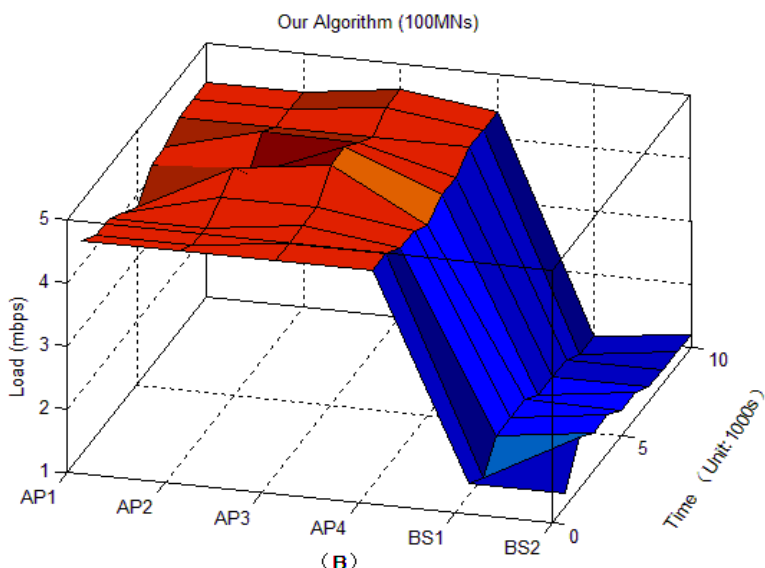

Figure 4. Load status for the our algorithm, when there are $100 \mathrm{MNs}$

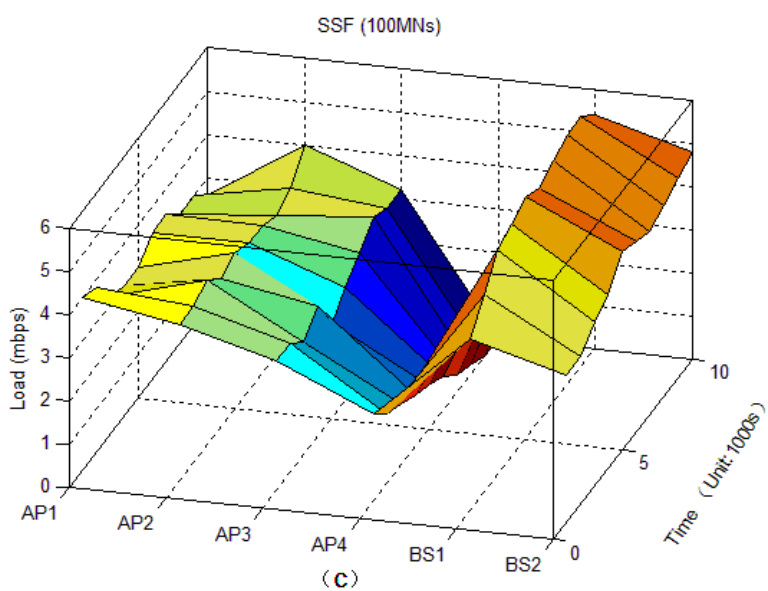

Figure 5. Load status for the SSF algorithm, when there are $100 \mathrm{MNs}$

In figure. 3 to 5, we plot the overall load at each AP and each BS versus the simulation time for the first test case with $100 \mathrm{MNs}$ active in the test area. Similarly, the overall loads for the second test case with 150 active MNs are plotted in figure. 6 to 8 . These figures show how the load is distributed among heterogeneous networks. In different cases, the same result occurs. Our algorithm achieves the best performance in terms

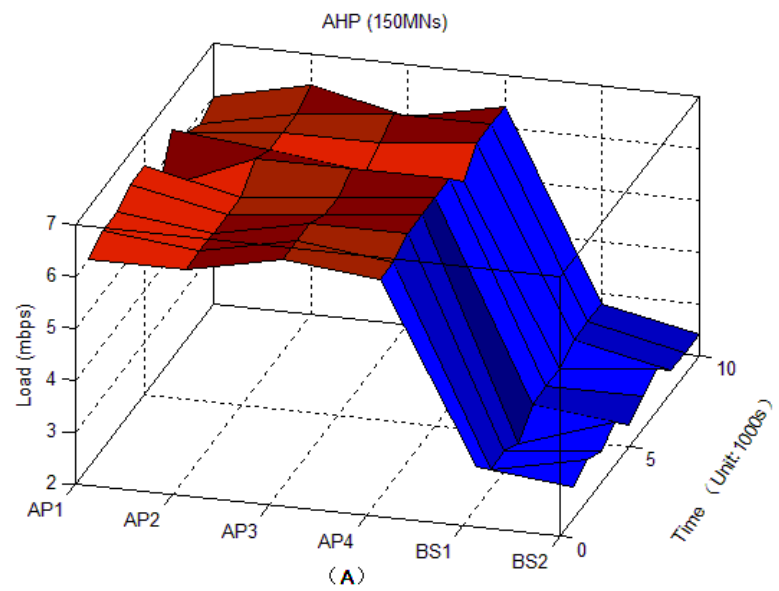

Figure 6. Load status for the AHP algorithm, when there are $150 \mathrm{MNs}$

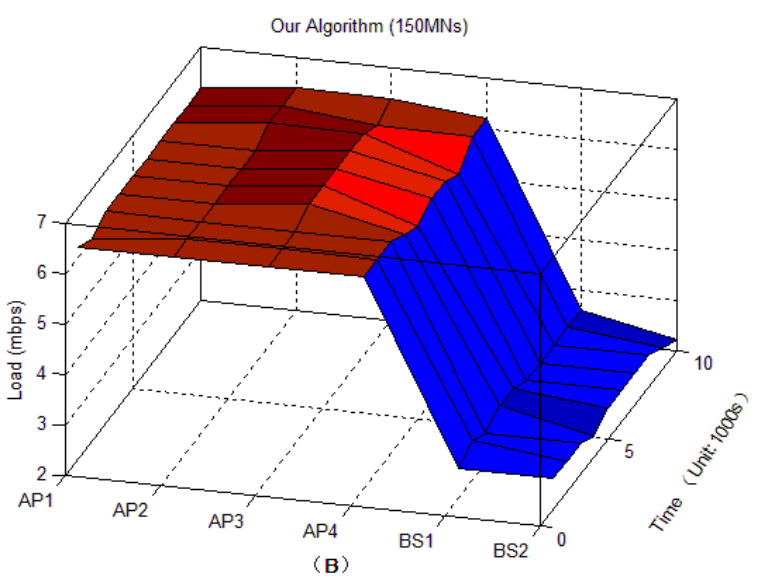

Figure 7. Load status for the Our algorithm, when there are $150 \mathrm{MNs}$

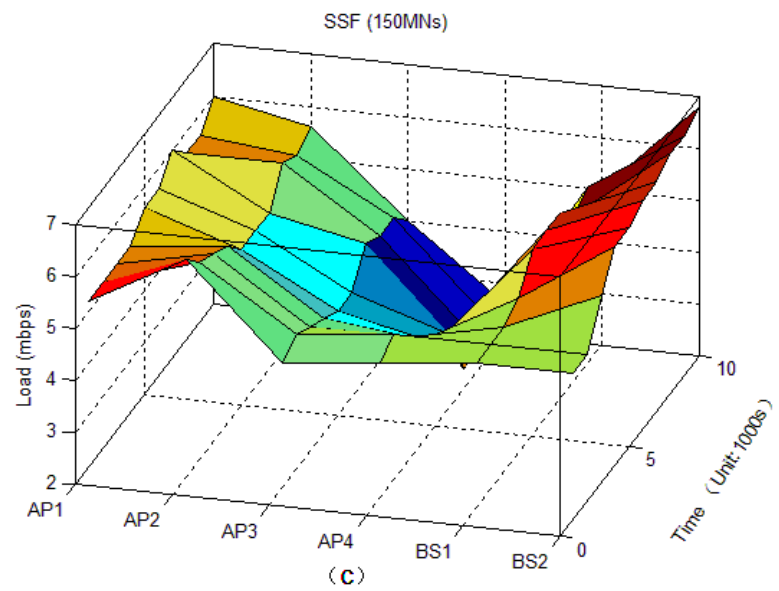

Figure 8. Load status for the SSF algorithm, when there are $150 \mathrm{MNs}$

of the distribution of load, the AHP algorithm comes second and the SSF method performs worst. 
From figure.9, it is easy to find out that when the quantities of connected MNs increase, the AHP algorithm and our algorithm both get a better load balance. However, the performance of SSF may perform even worse. In all case, our

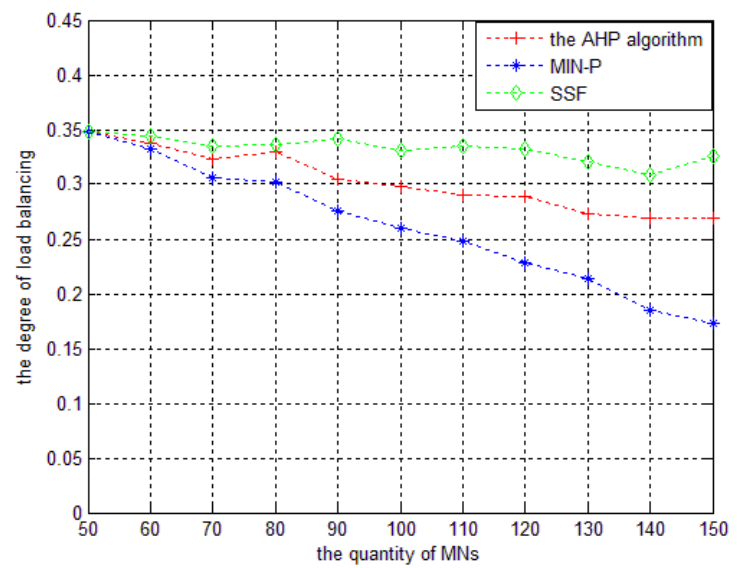

Figure 9. Load status for the SSF algorithm, when there are $150 \mathrm{MNs}$

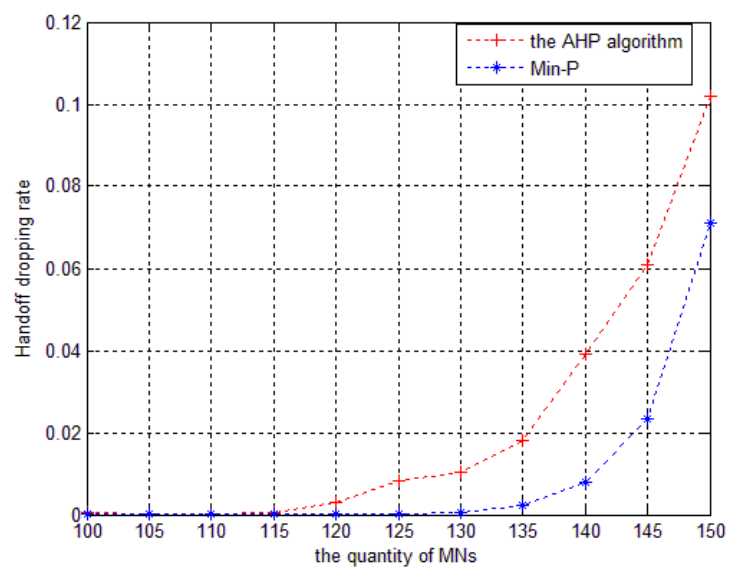

Figure 10. The maximum of handoff dropping probability in networks when MNs increase

method gets better performance than the AHP algorithm and the SSF method.

Figure 10 shows the maximum of handoff dropping probability in the six candidate networks when classifying the traditional AHP algorithm and our algorithm; the quantities of MNs increase from 100 to 150. The red plots in Figures 10 correspond to the traditional AHP algorithm and the blue plots correspond to ours. It could be seen that the maximum of handoff dropping probability in the six candidate networks increases monotonically when the connected MNs of the whole simulation area increase, which comply with the real situation. And it is also visible that when the quantity of connected MNs and the loads of the simulation area vary, our method always shows the improvement of reducing the maximum of handoff dropping probability over the traditional AHP algorithm.
More specially, in figure 11 , we can also evaluate the multiples of reducing the max handoff dropping probability the between traditional AHP algorithm and our algorithm. The results show that our algorithm always outperforms the traditional AHP algorithm though the ratio of reducing handoff

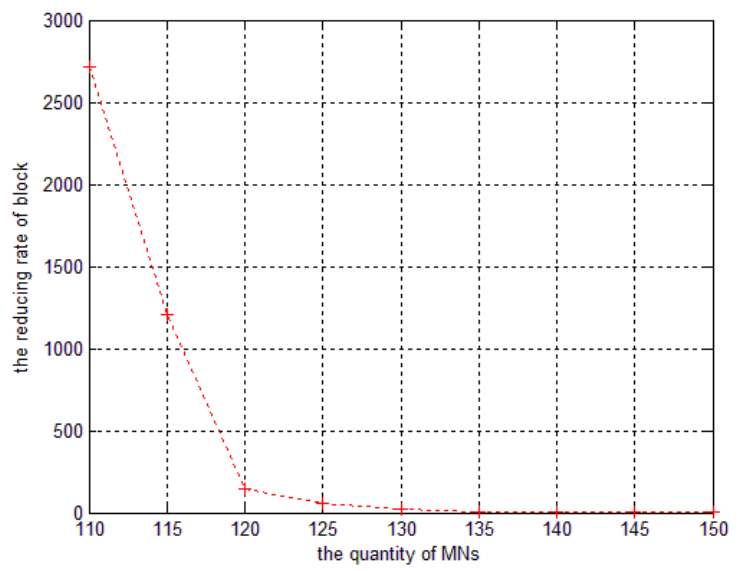

Figure 11. Ratio of reducing the handoff dropping probability between the AHP algorithm and our algorithm

probability decreases sharply when the loads of the whole area increase.

\section{CONCLUSion}

In this paper, we focus on the metrics selection for the VHD and propose an index named new handoff blocking probability to evaluate the network performance. We also formulate the network architecture by adding VHDS (including VHDT and CVHDC) to manage wireless resources effectively. Then a smart vertical handoff decision algorithm is proposed based on queuing theory. The performance results based on detailed simulations show that our algorithm performs much better than the conventional AHP algorithm and SFF method. The proposed algorithm not only ensures the accuracy in network selectivity but also reaches the balance of load distribution over APs and BSs and effectively reduces the probability of handoff dropping.

\section{ACKNOWLEDGMENT}

This work was supported by NSFC (No.61101106) and the Fundamental Research Funds for the Central Universities (No.2011RC0107, No.2013RC0120).

\section{REFERENCES}

[1] Universal mobile telecommunications system (UMTS); evolution of 3GPP system, ETSI TR 121902 V9.0.0 2010.

[2] E. Stevens-Navarro, Y. Lin and V. W. S. Wong, "An MDP-based vertical handoff decision algorithm for heterogeneous wireless networks," IEEE Transactions on Vehicular Technology, vol.57, pp. 1243-1254, 2008.

[3] Liu, X., et al., "An improved consumer goods market model-based vertical handoff decision algorithm". IEICE Electronics Express, 9(4): pp. $234-239,2012$. 
[4] M. Liu, Z. C. Li, X. B. Guo, E. Dutkiewicz and D. Zhang, "Performance evaluation of vertical handoff decision algorithms in heterogeneous wireless networks," in IEEE GLOBECOM'06.2006, pp. 1-5.

[5] M. Kassar, B. Kervella and G. Pujolle, "An overview of vertical handover decision strategies in heterogeneous wireless networks," Computer Communications, vol.31, pp. 2607-2620, 2008.

[6] A. Calhan and C. Ceken, "An Optimum Vertical Handoff Decision Algorithm Based on Adaptive Fuzzy Logic and Genetic Algorithm," Wireless Personal Communications, vol.64, pp. 647-664, 2012.

[7] P. Zhang, W. Zhou, B. Xie, and J. Song, "A novel network selection mechanism in an integrated WLAN and UMTS environment using AHP and modified GRA," in IEEE IC-NIDC, 2010, pp. 104-109.

[8] S. F. Zhu, F. Liu, Z. Y. Chai, Y. T. Qin and J. S. Wu, "Simple harmonic oscillator immune optimization algorithm for solving vertical handoff decision problem in heterogeneous wireless network," Acta Physica Sinica, vol.61, 2012.

[9] Y. S. Guo, G. Z. Tan, A. Libda and L. Q. Ma, "A QoS-aware vertical handoff algorithm based on predictive network information," Journal Of Central South University, vol.19, pp. 2187-2193, 2012.

[10] E. Arun and R. S. Moni, "Optimization of Vertical Handoff Decision Algorithm for Wireless Networks," International Journal Of Computers Communications \& Control, vol.7, 2012.

[11] S. Lee, K. Sriram, K. Kim, Y. H. Kim and N. Golmie, "Vertical handoff decision algorithms for providing optimized performance in heterogeneous wireless networks," IEEE Transactions on Vehicular Technology, vol.58, pp. 865-881, 2009.

[12] Standard and Metropolitan Area Networks: Media Independent Handover Services, IEEE Std. 802.21, Jan. 2006.

[13] D. J. He, C. X. Chi, S. M. Chan, C. Chen, J. J. Bu, and M. J. Yin, "A Simple and Robust Vertical Handoff Algorithm for Heterogeneous Wireless Mobile Networks," Wireless Personal Communications, vol. 59, pp. 361-373, 2011.

[14] C. Oliveira, J. B. Kim and T. Suda, "Adaptive bandwidth reservation scheme for high-speed multimedia wireless networks," IEEE Journal on Selected Areas in Communications, vol.16, pp. 858-874, 1998.

[15] C. Gu, M. Song, Y. Zhang, L. Wang, and J. Song, "Novel network selection mechanism using AHP and enhanced GA," in CNSR '09.2009, pp. 397-401.

Yong Sun (M'12) Beijing University of Posts and Telecommunications information and communication engineering,

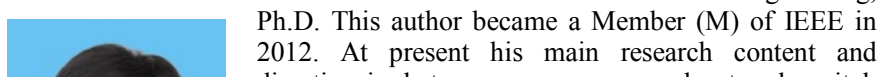
direction is: heterogeneous converged network switch decision theory and control method of research, multimedia collaborative theory and its application in broadband wireless access technology research. 